\title{
High-Resolution MRI Evaluation of Neonatal Brachial Plexus Palsy: A Promising Alternative to Traditional CT Myelography
}

\author{
D. Somashekar, L.J.S. Yang, M. Ibrahim, and H.A. Parmar
}

\begin{abstract}
BACKGROUND AND PURPOSE: Despite recent improvements in perinatal care, the incidence of neonatal brachial plexus palsy remains relatively common. CT myelography is currently considered to be the optimal imaging technique for evaluating nerve root integrity. Recent improvements in MR imaging techniques have made it an attractive alternative to evaluate nerve root avulsions (preganglionic injuries). We aim to demonstrate utility of MR imaging in the evaluation of normal and avulsed spinal nerve roots.
\end{abstract}

MATERIALS AND METHODS: All study patients with clinically diagnosed neonatal brachial plexus palsy underwent MR imaging by use of a high-resolution, heavily T2-weighted (driven equilibrium) sequence. MR imaging findings were reviewed for presence of nerve root avulsion from $\mathrm{C} 5-\mathrm{Tl}$ and for presence of pseudomeningocele. The intraoperative findings were reviewed and compared with the preoperative MR imaging findings.

RESULTS: Thirteen patients ( 9 male, 4 female) underwent MR imaging; 6 patients underwent nerve reconstruction surgery, during which a total of 19 nerve roots were evaluated. Eight avulsions were noted at surgery and in the remainder, the nerve injury was more distal (rupture/postganglionic injury). Six of the 8 nerve root avulsions identified at surgery were at C5-6 level, whereas 1 nerve root avulsion was identified at C7 and C8 levels, respectively. The overall sensitivity and specificity of MR imaging for nerve root avulsions was $75 \%$ and $82 \%$, respectively.

CONCLUSIONS: Our preliminary results demonstrate that high-resolution MR imaging offers an excellent alternative to CT myelography for the evaluation of neonatal brachial plexus palsy with similar sensitivity compared with CT myelography.

ABBREVIATIONS: NBPP $=$ neonatal brachial plexus palsy; $\mathrm{CTM}=\mathrm{CT}$ myelogram

N eonatal brachial plexus palsy (NBPP) results from insult to the brachial plexus during the perinatal period. ${ }^{1} \mathrm{NBPP}$ can result when the upper shoulder of the infant becomes blocked by the pubic symphysis of the mother. ${ }^{2,3}$ Nerve injury can occur anywhere along the brachial plexus but generally occurs in the supraclavicular brachial plexus at the nerve roots/trunks levels, resulting in varied neurologic deficits. Damage to the nerve roots arising from the ventral aspect of the spinal cord results in motor function disability. The most common lesions occur within the C5 and C6 spinal nerves ( $80 \%$ of patients), with a smaller group of patients having more extensive lesions ranging from $\mathrm{C} 5-\mathrm{C} 7$ and

Received August 20, 2013; accepted after revision October 8.

From the Departments of Radiology (D.S., M.I., H.A.P.) and Neurosurgery (L.J.S.Y.), University of Michigan Hospital, Ann Arbor, Michigan.

Paper previously presented at: Annual Meeting of the American Society of Neuroradiology, May 18-23, 2013; San Diego, California.

Please address correspondence to Hemant A. Parmar, MD, Department of Radiology, University of Michigan, Taubman Center/B1/132 F, 1500 E Medical Center Dr, Ann Arbor, MI 48105; e-mail: parurad@hotmail.com

http://dx.doi.org/10.3174/ajnr.A3820 from C5-T1 (pan-plexopathy). ${ }^{1,3}$ Collectively, the clinical presentation resulting from these lesions is referred to as NBPP.

NBPP occurs with an incidence of up to 3 per 1000 live births. ${ }^{1}$ The most severe forms of injury result from complete axonal disruption (neurotmesis or severe axonotmesis), either at the level of the proximal nerve roots or trunks of the brachial plexus (ruptures, postganglionic injury), or when 1 or more of the spinal nerves of the brachial plexus are torn out of the spinal cord (root avulsion, preganglionic injury). ${ }^{4}$ In these cases, the likelihood of spontaneous recovery is low and surgical intervention is generally thought to be reasonable. ${ }^{5}$ Less severe injuries such as simple stretching of the nerves (neurapraxia) or rupture of a few axons (mild axonotmesis) can result in spontaneous functional recov$\operatorname{ery}^{3}$ (Fig 1). The clinical treatment of patients with NBPP can be difficult and depends on the specific type of lesion involved. Early on, it is often difficult to characterize the lesion type because patients may clinically present with similar apparent deficiencies regardless of the levels involved. This presents a diagnostic and management dilemma because patients with neurapraxia/mild 
A

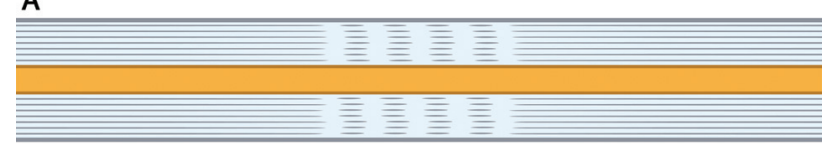

B

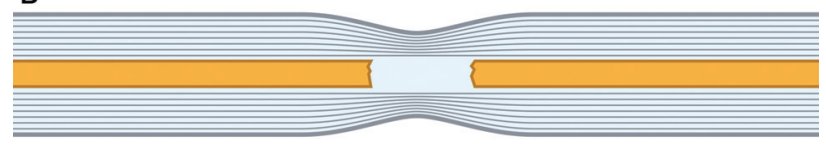

C

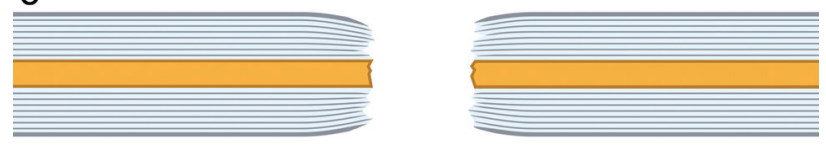

FIG 1. Schematic diagram showing different patterns of injuries affecting the nerve, including neuropraxia (A), mild axonotmesis (B), and neurotmesis (C).

axonotmesis will demonstrate spontaneous recovery over time, whereas the effectiveness of surgical intervention for neurotmesis or nerve root avulsion decreases with time. The typical practice at this time is to allow the patient a 3-month period in which to exhibit spontaneous recovery. ${ }^{4,5}$ If recovery does not occur or is incomplete, further evaluation is recommended to determine the extent of injury. Perhaps there is a role for imaging earlier after birth because patients with minor injuries could be given a more favorable prognosis without the waiting period, at the admitted increased medical costs. However, it is possible that early imaging can save medical costs downstream by identifying patients who do not need more extensive follow-up and evaluation in the future.

Although direct surgical exploration may be considered the reference standard for lesion characterization, it carries significant morbidity and would require laminectomy to observe the intradural nerve roots. For this reason, CT myelography (CTM) and electrodiagnostic studies have been used as less invasive techniques and comprise the standard preoperative assessment for establishing preganglionic nerve root avulsion and postganglionic nerve ruptures in neonatal, pediatric, and adult populations. ${ }^{4,6}$ CTM is most useful for detection of root avulsions ( $72 \%$ sensitivity), whereas electrodiagnostic studies are best at detecting postganglionic nerve ruptures, especially in the upper plexus $\left(93 \%\right.$ sensitivity). ${ }^{6}$ These 2 tests are generally used in combination with one another to provide the neurosurgeon with supplemental preoperative information. ${ }^{6}$

Although CTM is currently the most widely used imaging method for evaluating nerve root avulsion, there are drawbacks. It requires an invasive lumbar puncture, instillation of iodinated contrast into the thecal sac, and the use of radiation, all of which carry unfavorable risks, particularly within the infant and pediatric populations. Nevertheless, CTM continues to be recommended in every preoperative assessment for NBPP at many specialty centers. ${ }^{4,6}$ Recent improvements in MR imaging techniques have made MR imaging an attractive alternative to conventional CT. MR imaging is noninvasive, does not require the use of intrathecal contrast, and does not use ionizing radiation. This tech- nique, if effective at diagnosing nerve root avulsion, can emerge as an alternative technique to CTM in the pediatric population. To date, however, there are only a few reports contained in the literature examining the utility of MR imaging for nerve root avulsions and none looking specifically at NBPP. ${ }^{7-11}$ The reports contain scant imaging examples of nerve root avulsion, and many of the images are not convincingly diagnostic. ${ }^{12}$ Most of the reports focus on the use of a heavily T2-weighted 3D sequence, referred to under various names on the basis of the specific manufacturer, such as 3D CISS (constructive interference in steady state), 3D True-FISP (fast imaging with steady-state precession), FIESTA (fast imaging employing steadystate acquisition), and DRIVE (driven equilibrium) sequences. ${ }^{7}$ The end goal of these sequences is the same: to create a sequence with very high CSF-to-tissue contrast with elimination of pulsation artifact, to optimally visualize the exiting cervicothoracic nerve roots. ${ }^{13}$ Until now, however, there are no studies that unequivocally and consistently demonstrate high-quality images of nerve root avulsion. Some propose that it lacks the requisite spatial resolution to provide the neurosurgeon with necessary diagnostic information, ${ }^{14}$ though more recent advances in high-resolution $3 \mathrm{~T}$ MR challenge this proposition. ${ }^{15}$ Our aim was to use high-resolution MR imaging in evaluation of ventral nerve root avulsions in NBPP and to demonstrate that it is an excellent noninvasive and nonirradiating alternative to CTM.

\section{MATERIALS AND METHODS}

Institutional review board approval was obtained, and patient consent was waived for this Health Insurance Portability and Accountability Act-compliant prospective study. Patients were referred to our institution for evaluation of NBPP. All patients were given an obligatory observation period of 3-4 months to assess for spontaneous recovery. If clinical improvement was not forthcoming, they were referred for additional evaluation including MR imaging. MR imaging examination was performed on a $3 \mathrm{~T}$ magnet (Ingenia; Philips Healthcare, Best, the Netherlands). We used a high-resolution 3D T2 DRIVE sequence with TR/TE/ of $1500 / 100 \mathrm{~ms}$, TSE factor of 40 , uniform voxel size of $0.6 \mathrm{~mm}$ with field of view of $80 \mathrm{~mm}$, and reconstruction matrix of $320 \times 320$. A sensitivity encoding factor (parallel imaging) of 1.6 was used. Total scan time for this sequence was 8 minutes, 43 seconds. Sagittal and coronal reformatted images on both right and left sides were obtained in all patients and reviewed. All imaging studies were independently reviewed by 2 board-certified and pediatric neuroradiology-trained radiologists. Findings of presence or absence of ventral nerve root avulsions were recorded by consensus. At the time of writing, 6 of the 13 patients had proceeded to surgery. The findings at the time of surgery were recorded. The initial radiologic diagnoses were then compared with the surgical findings for any discrepancy (Table).

\section{RESULTS}

Thirteen patients ( 9 male, 4 female) with clinically diagnosed NBPP underwent MR imaging evaluation. Average age at the time of imaging was 6 months. MR imaging was successful and able to visualize the individual ventral and dorsal nerve roots in all patients (Fig $2 A-C$ ). We used axial images as primary images for our analysis; sagittal and coronal reformatted images complemented 


\begin{tabular}{|c|c|c|c|c|c|c|c|c|c|c|c|}
\hline \multicolumn{12}{|c|}{ Cervical Level } \\
\hline \multirow[b]{2}{*}{ Patient } & \multirow[b]{2}{*}{ Affected Side } & \multicolumn{2}{|c|}{ C5 } & \multicolumn{2}{|c|}{$\mathrm{C} 6$} & \multicolumn{2}{|c|}{$\mathrm{C7}$} & \multicolumn{2}{|c|}{$\mathrm{C} 8$} & \multicolumn{2}{|c|}{$\mathrm{T1}$} \\
\hline & & MRI & Surgery & MRI & Surgery & MRI & Surgery & MRI & Surgery & MRI & Surgery \\
\hline 1 & Right & $\mathrm{N}$ & $\mathrm{N}$ & $\mathrm{N}$ & $\mathrm{N}$ & $\mathrm{N}$ & $N$ & $\mathrm{~N}$ & $\mathrm{~N} / \mathrm{A}$ & $\mathrm{N}$ & $\mathrm{N} / \mathrm{A}$ \\
\hline 2 & Left & $\mathrm{N}$ & $\mathrm{N}$ & $\mathrm{N}$ & $A$ & $\mathrm{~N}$ & $\mathrm{~N}$ & $\mathrm{~N}$ & $\mathrm{~N} / \mathrm{A}$ & $\mathrm{N}$ & $\mathrm{N} / \mathrm{A}$ \\
\hline 3 & Right & $A$ & $A$ & $A$ & A & $A$ & $\mathrm{~N}$ & $A$ & $\mathrm{~N} / \mathrm{A}$ & $\mathrm{N}$ & $\mathrm{N} / \mathrm{A}$ \\
\hline 4 & Left & $\mathrm{N}$ & $\mathrm{N}$ & $\mathrm{N}$ & $A$ & $\mathrm{~N}$ & $\mathrm{~N}$ & $\mathrm{~N}$ & N/A & $\mathrm{N}$ & N/A \\
\hline 5 & Right & $\mathrm{N}$ & $\mathrm{N}$ & $A$ & A & $A$ & $A$ & $A$ & $A$ & $\mathrm{~N}$ & $N$ \\
\hline 6 & Right & $\mathrm{N}$ & $\mathrm{N}$ & $A$ & A & $A$ & $N$ & $N$ & $\mathrm{~N} / \mathrm{A}$ & $N$ & $\mathrm{~N} / \mathrm{A}$ \\
\hline
\end{tabular}

Note:-N indicates normal; A, avulsion.
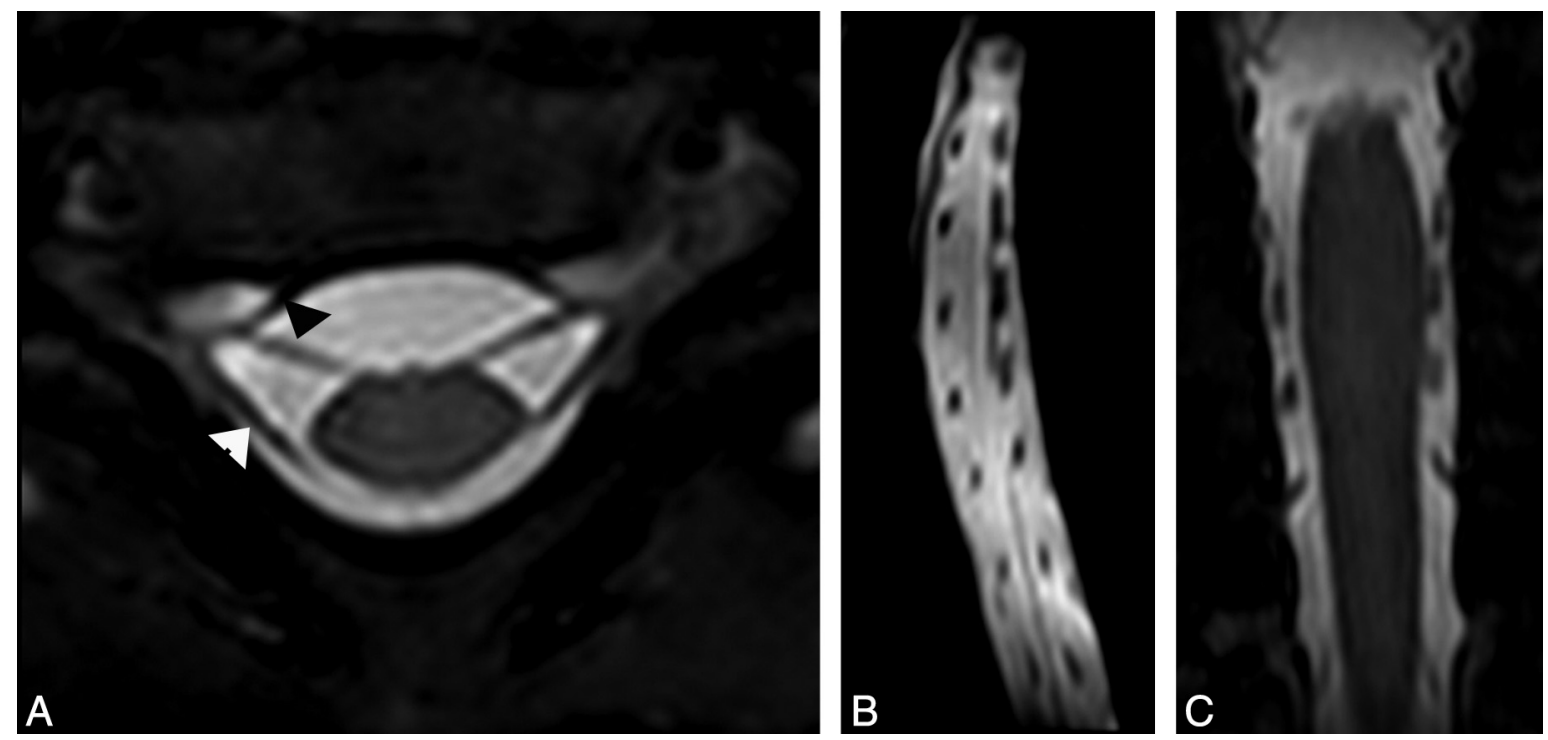

FIG 2. A, Axial high-resolution MR imaging in a 6-month-old girl with clinically suspected right-sided brachial plexus palsy showing intact right ventral (arrowhead) and dorsal nerve roots on both sides. Compare with normal left-sided nerve roots. Sagittal (B) and (C) coronal reformatted images from the axial data again show normal ventral and dorsal nerve roots at each vertebral level.

the axial images by showing multiple nerve roots at the same time. Of the 13 patients, 6 underwent brachial plexus exploration and nerve reconstruction, from which a total of 19 nerve roots were evaluated. At surgery, 8 ventral nerve root avulsions were noted. Overall, MR imaging was $75 \%$ sensitive and $83 \%$ specific in the preoperative detection of these ventral nerve root avulsions. The positive and negative predictive values were also $75 \%$ and $83 \%$, respectively. A more in-depth, level-by-level analysis demonstrated that 6 of 8 surgically confirmed avulsions occurred at the C5 and C6 levels (Fig 3A-F). At C5, there was 1 avulsion, which was correctly identified by means of MR imaging ( $100 \%$ sensitive and specific). At C6, there were 5 surgically proved avulsions. Of these, MR imaging was successful in detecting 3 of the lesions (60\% sensitive, $100 \%$ specific). Interestingly, the remaining 2 avulsions that were not detected did not have any evidence of associated pseudomeningocele, either on imaging or at surgery. The C6 level nerve roots were reanalyzed again in light of surgical data, but no obvious technical issues on MR images were noticed to explain this discordance. At C7, there was 1 surgically confirmed avulsion that was detected by MR imaging; however, there were 2 additional false-positive C7 avulsions (100\% sensitive, $60 \%$ specific, $33 \%$ positive predictive value). MR imaging correctly identified 1 avulsion at C8. An additional C8 avulsion was detected, but this level was not explored surgically and thus there was no confirmation. At the T1 level,
MR imaging did not detect any abnormalities. Only 1 of these T1 levels was surgically confirmed to be normal, whereas none of the other T1 roots were explored, and thus confirmation of whether these roots were actually intact was not possible. MR imaging detected 5 pseudomeningoceles (Fig 4) occurring in 3 patients, all of which were associated with nerve root avulsions. Of note, no MR imaging avulsions were identified in the absence of a pseudomeningocele. Interestingly, however, there were 2 surgically confirmed C6 avulsions. In both of these cases, there was no evidence of pseudomeningocele.

\section{DISCUSSION}

NBPP includes a wide array of injuries, primarily to the exiting nerve roots and trunks of the brachial plexus. Identification of avulsion (preganglionic) injuries is critical for maximizing outcomes by early surgical intervention and for operative planning: the nerve reconstruction strategy for avulsion injuries significantly differs from that of rupture (postganglionic injuries). In the case of ruptured nerve roots or trunks, autologous nerve grafting is usually used. A harvested nerve, often the sural nerve, is used to bridge the gap between the disrupted elements of the brachial plexus. $^{3}$ This provides a physical pathway as well as neurotrophic factors to stimulate axonal outgrowth. This surgery is often accomplished by means of a supraclavicular approach. ${ }^{5}$ Brachial plexus root avulsion provides an additional challenge because 

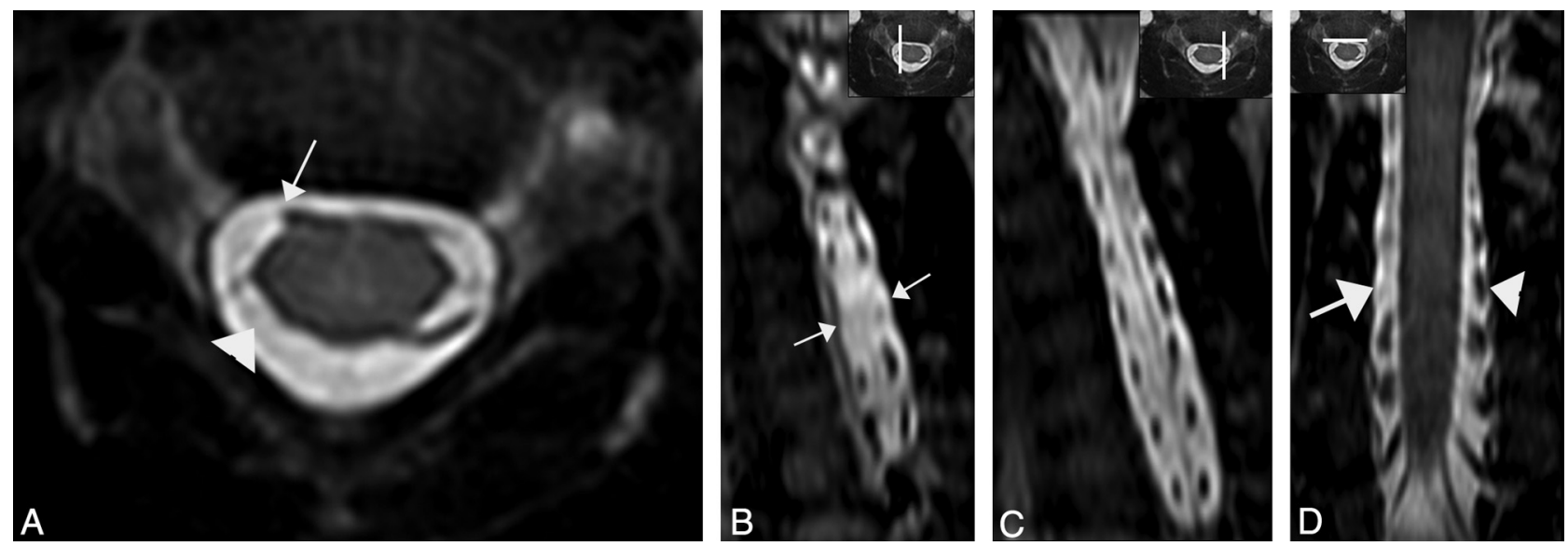

FIG 3. A, Axial high-resolution MR imaging in a 5-month-old girl with clinically suspected right-sided brachial plexus palsy shows avulsion injury of the right C5 ventral nerve root (arrow). The right dorsal nerve root is also avulsed (arrowhead). Sagittal (B) reformatted images on the right side show absent ventral and dorsal C5 nerve roots (arrows), compared with the normal nerve roots on left side (C). Coronal reformatted image (D) at the level of ventral nerve roots show absent right C5 ventral nerve root from avulsion (arrow), compared with the normal left-sided nerve root (arrowhead).

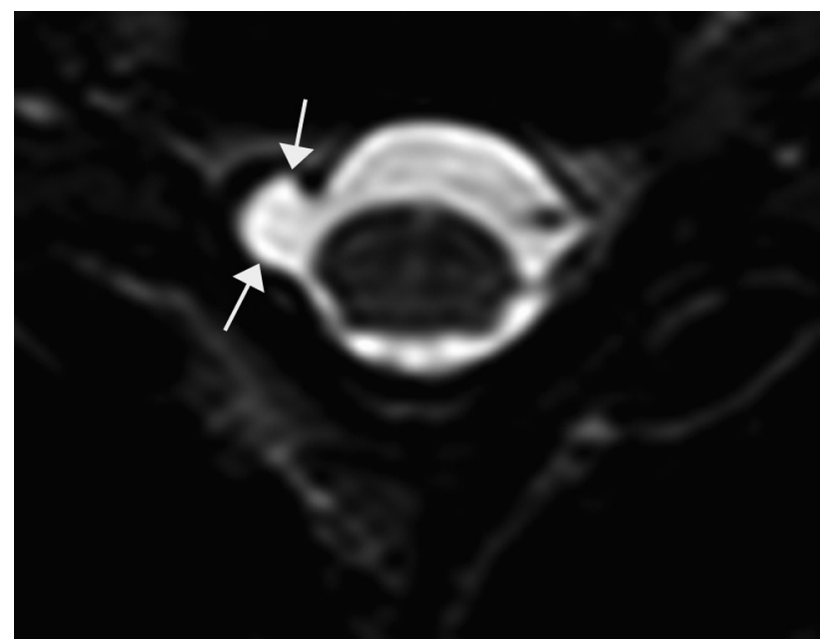

FIG 4. Axial high-resolution MR imaging in a 4-month-old boy with clinically suspected right-sided brachial plexus palsy shows a pseudomeningocele at right C5-6 level (arrow). Note absent nerve roots on right side suggestive of nerve root avulsion injury. Compare with normal ventral and dorsal nerve roots on the left side.

currently there is no feasible way in which to reattach the avulsed root to the spinal cord. The solution is a nerve transfer, in which an extra-plexus exiting nerve is cut and coapted to the denervated brachial plexus terminal nerve. ${ }^{3}$

In the present study, we evaluated a total of 13 patients by use of high-resolution MR imaging, of which 6 went on to surgery for confirmation and repair. In terms of overall performance, MR imaging demonstrated a sensitivity of $75 \%$ and specificity of $83 \%$, both of which are comparable to the results published on CTM. Vanderhave et $\mathrm{al}^{6}$ found the sensitivity of CTM as compared with surgical exploration to be $50 \%$ at levels C5 and C6, 83.3\% at C7, and $75 \%$ at C8 and T1. There were 2 instances in which MR imaging failed to detect nerve root avulsion, both at the C6 level in different patients. These avulsions were detected at surgery, and these patients had no other roots that were avulsed. Curiously, no secondary findings such as pseudomeningocele were identified, either on imaging or at surgery. Isolated root avulsion without such secondary findings of trauma is quite unusual, and no satisfactory anatomic explanation exists; perhaps there was scarring of the pseudomeningocele in the interval from time of injury to the time of imaging. Nonetheless, these 2 cases provide excellent examples in which nerve root avulsions can exist at surgery without the presence of pseudomeningocele formation. Therefore, it is imperative that MR and CT emphasize the imaging of actual nerve roots and that the neuroradiologist should not rely solely on the detection of pseudomeningoceles to confirm or discount the existence of a root avulsion. Our results demonstrate that MR imaging offers an excellent alternative to CT myelography in the evaluation of complete brachial plexus nerve root avulsion(s). The high-resolution MR imaging technique provides unambiguous visualization of intact nerve roots and accurate assessment of nerve root avulsions. MR imaging is also able to clearly show dorsal and ventral nerve roots in all 3 planes. It is important to differentiate the dorsal and ventral nerve roots and assess whether they are intact or avulsed because an intact dorsal nerve root can be used as a donor for an avulsed ventral nerve root by the neurosurgeon.

There are limitations to this study. First and foremost, the sample size is small, with 13 patients included in the study, only 6 of whom went on to surgery. Nevertheless, this is the first study of its kind to compare MR imaging findings with the reference standard of surgical exploration in the setting of NBPP. Another limitation stems from the lack of comparison CTM within this study group. Ideally, CTM and MR imaging would have been obtained in all patients to allow for a one-to-one comparison of the accuracy of the tests, but, obviously, given the ethical considerations, this was not deemed possible. Thus, the sensitivities and specificities for MR imaging were compared with those of CTM in the already published literature. The third limitation is that with the exception of 1 patient, the remaining C8 and T1 levels were never surgically observed. This in part had to do with the alternate, more in-depth surgical approach necessary to access these levels. This resulted in $1 \mathrm{C} 8$ avulsion being detected on MR but never undergoing surgical confirmation. This was an isolated, nonsurgically confirmed avulsion. All other avulsions were surgically confirmed or refuted. 


\section{CONCLUSIONS}

By prospectively examining 13 patients with clinically diagnosed NBPP, we have demonstrated the potential utility of MR imaging for providing reliable preoperative diagnoses of the type and extent of injury. It has proven value and has supplanted the use of CTM at our institution. Given that it is both noninvasive and nonirradiating while still providing all of the diagnostic information necessary to aid our neurosurgical colleagues, MR imaging should be the recommended technique in evaluating nerve root avulsion injuries in patients with NBPP.

\section{ACKNOWLEDGMENTS}

The authors would like to thank Leslie Burrell of the Radiology Media Division at University of Michigan for the illustration used in Fig 1.

Disclosures: Lynda Yang-UNRELATED: Consultancy: Chair of the Neurological Devices Advisory Committee at the CDRH division of the FDA.

\section{REFERENCES}

1. Doumouchtsis SK, Arulkumaran S. Are all brachial plexus injuries caused by shoulder dystocia? Obstet Gynecol Surv 2009;64:615-23

2. Alfonso DT. Causes of neonatal brachial plexus palsy. Bull NYU Hosp Jt Dis 2011;69:11-16

3. Malessy MJ, Pondaag W. Nerve surgery for neonatal brachial plexus palsy. J Pediatr Rehabil Med 2011;4:141-48

4. Steens SC, Pondaag W, Malessy MJ, et al. Obstetric brachial plexus lesions: CT myelography. Radiology 2011;259:508-15

5. Chung KC, Yang LJS, McGillicuddy JE. Practical Management of Pediatric and Adult Brachial Plexus Palsies. Philadelphia: Elsevier Health; 2011:35-46
6. Vanderhave KL, Bovid K, Alpert $\mathrm{H}$, et al. Utility of electrodiagnostic testing and computed tomography myelography in the preoperative evaluation of neonatal brachial plexus palsy. J Neurosurg Pediatr 2012;9:283-89

7. Vargas MI, Viallon M, Nguyen D, et al. New approaches in imaging of the brachial plexus. Eur J Radiol 2010;74:403-10

8. Du R, Auguste KI, Chin CT, et al. Magnetic resonance neurography for the evaluation of peripheral nerve, brachial plexus, and nerve root disorders. J Neurosurg 2010;112:362-71

9. van Ouwerkerk WJ, Strijers RL, Barkhof F, et al. Detection of root avulsion in the dominant $\mathrm{C} 7$ obstetric brachial plexus lesion: experience with three-dimensional constructive interference in steadystate magnetic resonance imaging and electrophysiology. Neurosurgery 2005;57:930-40

10. Doi K, Otsuka K, Okamoto $\mathrm{Y}$, et al. Cervical nerve root avulsion in brachial plexus injuries: magnetic resonance imaging classification and comparison with myelography and computerized tomography myelography. J Neurosurg 2002;96:277-84

11. Hayes CE, Tsuruda JS, Mathis CM, et al. Brachail plexus: MR imaging with a dedicated phased array of surface coils. Radiology 1997;203:286-89

12. Smith AB, Gupta N, Strober J, et al. Magnetic resonance neurography in children with birth-related brachial plexus injury. Pediatr Radiol 2008;38:159-63

13. Hingwala D, Chatterjee S, Kesavadas C, et al. Applications of 3D CISS sequence for problem solving in neuroimaging. Indian J Radiol Imaging 2011;21:90-97

14. Yoshikawa T, Hayashi N, Yamamoto S, et al. Brachial plexus injury: clinical manifestations, conventional imaging findings, and the latest imaging techniques. Radiographics 2006;26:S133-43

15. Chhabra A, Thawait GK, Soltados T, et al. High-resolution 3T MR neurography of the brachial plexus and its branches, with emphasis on 3D imaging. AJNR Am J Neuroradiol 2013;34:486-97 